FORMATION Formation emploi

Revue française de sciences sociales

$105 \mid$ janvier-mars 2009

Pêle-mêle

\title{
La place des diplômes dans la carrière des ouvriers de la filière automobile
}

Die Bedeutung von Abschlusszeugnissen im beruflichen Werdegang von

Arbeitern der Automobilbranche

The role of diplomas in automobile worker careers

El lugar de los diplomas en la carrera de los obreros del sector automotriz

\section{Armelle Gorgeu et René Mathieu}

\section{(Q) OpenEdition}

Journals

Édition électronique

URL : http://journals.openedition.org/formationemploi/1819

DOI : 10.4000/formationemploi.1819

ISSN : 2107-0946

Éditeur

La Documentation française

Édition imprimée

Date de publication : 1 mars 2009

Pagination : $37-51$

ISSN : 0759-6340

Référence électronique

Armelle Gorgeu et René Mathieu, « La place des diplômes dans la carrière des ouvriers de la filière automobile », Formation emploi [En ligne], 105 | janvier-mars 2009, mis en ligne le 01 mars 2011. consulté le 30 octobre 2020. URL : http://journals.openedition.org/formationemploi/1819 ; DOI : https://doi.org/10.4000/formationemploi.1819 


\section{Profession}

\section{La place des diplômes dans la carrière des ouvriers de la filière automobile}

Armelle Gorgeu et René Mathieu*

Les diplômes et certificats sont, pour les employeurs de la filière automobile étudiée ici, des "réducteurs d'incertitude", mais rien ne garantit que cela leur confère de la valeur. Les certifications ne sont pas reconnues dans les conventions collectives, et les coefficients de classification correspondant aux niveaux de diplômes ne sont que rarement appliqués pour les ouvriers.

Le diplôme est devenu «un bien commun», le détenir devient indispensable pour accéder à l'emploi. Le rôle primordial joué par le diplôme pour les débutants est récent, puisque les études du Céreq, dans les années 80 , signalaient que le fait d'avoir suivi un cursus de formation pouvait compenser l'absence de diplômes. «Désormais, même quand la conjoncture de l'emploi est favorable (...) l'écart entre les diplômés et les non diplômés est important» (Maillard, 2006a, p. 51). Ce résultat concerne tous les jeunes, quel que soit le poste occupé, y compris les ouvriers. D'après les chiffres de l'enquête " Génération 98 » du Céreq, en 2003, $84 \%$ des jeunes sortis du système scolaire en 1998 sont effectivement engagés dans l'activité professionnelle cinq ans plus tard, et un peu plus d'un sur cinq occupe un emploi d'ouvrier, ce qui peut paraître surprenant car la part des ouvriers diminue dans
* Armelle Gorgeu et René Mathieu sont chercheurs au laboratoire "Genre, Travail, Mobilités 》 IUMR 7183 , CNRS et universités Paris VIII et Paris X). Ils sont socioéconomistes. Leurs recherches portent sur l'organisation du travail, la gestion de l'emploi et de la main-d'œuvre et les conditions de travail dans l'industrie, et tout particulièrement dans la filière automobile, dont ils suivent l'évolution depuis une vingtaine d'années. Ils ont publié récemment: Les bacheliers dans les usines d'équipement automobile: Vers la déqualification? (avec Olivia Blum), CPC Document, 2005, $\mathrm{n}^{\circ} 1$; : Polyvalence, polycompétence ouvrières et intensification du travail : I'exemple de l'industrie automobile " (avec Michel Pialoux) in Askenavy et alii, Organisation et intensité du travail, Octarès, 2006 ; "L'action contrainte des directions d'usines, analyse de la gestion de l'emploi dans les usines de la filière automobile ", Économies et Sociétés, Série socio-économie du travail, $n^{\circ} 26,2005$ 
l'ensemble de la population active ${ }^{1}$. "Ainsi le groupe ouvrier, vieux par son histoire, s'avère-t-il jeune!» (Eckert, 2006, p. 31). Le renouvellement de la maind'œuvre ouvrière s'explique par les nombreux départs (licenciements, cessations d'activité par retraites anticipées et retraites), mais aussi par la reprise des recrutements de jeunes ouvriers ${ }^{2}$, à la fin des années 90, dans de nombreux secteurs industriels, notamment dans l'industrie automobile.

Dans un contexte de montée des niveaux de formation des jeunes (Merle, 2002; Baudelot, Leclercq, 2005) et de rajeunissement de la main-d'œuvre ouvrière, nous nous proposons ici d'analyser la place des diplômes dans la sélection et dans la promotion des ouvriers de l'industrie, en prenant comme exemple la filière automobile (les constructeurs et leurs fournisseurs).

Les enquêtes monographiques que nous menons depuis une vingtaine d'années sur la gestion de l'emploi et de la main-d'œuvre dans les usines de la filière automobile, notamment sur les procédures de recrutement des ouvriers ( $c f$. encadré 1), peuvent apparaître comme complémentaires aux enquêtes générationnelles du Céreq. La sélectivité à l'embauche des ouvriers de la filière automobile repose sur une succession de filtres, où les épreuves d'évaluation par batteries de tests et entretiens semblent aujourd'hui prioritaires. De façon formelle ou implicite, le diplôme occupe une place dans la procédure de recrutement, mais il se prête à maints usages. En fonction des stratégies mises en place par les entreprises, de leurs technologies et de leur organisation, mais aussi en fonction des emplois qu'elles proposent en production, le diplôme peut être envisagé essentiellement comme une certification du comportement, une garantie d'acquisition de connaissances générales - et dans ce cas peu importe le contenu technique de la formation - ou bien être reconnu pour ses spécificités techniques. Le niveau

\footnotetext{
${ }^{1}$ D'après le recensement de 1999 , il y aurait à peine plus de 7 millions d'ouvriers en emploi ou en recherche d'un emploi (ces statistiques ont un champ plus large que l'industrie) contre près de 8 millions d'employés sur un total de 26 millions d'actifs.

${ }^{2}$ Henri Eckert (2006) précise que d'après l'enquête Emploi réalisée par l'INSEE en 2002, un ouvrier occupé sur quatre a entre 15 et 29 ans, et que, parmi les jeunes hommes de 15 à 29 ans en emploi, un sur deux est ouvrier (ces statistiques ont un champ plus large que l'industrie).
}

du diplôme exigé ou souhaité est aussi à prendre en considération : pour un emploi comparable, il peut être différent d'une entreprise à l'autre, et au sein d'un même établissement industriel, il peut varier selon les moments, notamment en fonction de la conjoncture et des renouvellements des équipes dirigeantes. Le diplôme ne sert pas qu'au moment du recrutement, il peut également faciliter l'évolution professionnelle, être un pré-requis pour accéder à un emploi de qualification supérieure, d'ouvrier professionnel, de technicien ou de maîtrise. Mais comme le montre Nicolas Hatzfeld (2004), dans le cas des ouvriers sur les chaînes de Peugeot-Sochaux, l'imbrication entre le système de classification et de rémunération, l'organisation du travail et la situation économique et sociale d'une époque donnée conduit à un "grippage de la mobilité » qui, dans le cas de Peugeot-Sochaux, concerne d'abord les anciens ${ }^{3}$, mais qui, dans des usines fournisseurs où la maind'œuvre est plus jeune, peut s'appliquer à tous les ouvriers, quel que soit leur niveau de diplôme.

Les informations présentées dans cet article peuvent sembler appartenir à un passé révolu, car il n'est plus question aujourd'hui de recrutements d'ouvriers dans la filière automobile, compte tenu des évolutions récentes de l'emploi, des menaces de délocalisations d'activités et des perspectives incertaines qui en découlent. Néanmoins, avec les nombreux départs qui se profilent, de nouveaux besoins de personnel se feront sentir lors de la reprise, notamment d'intérimaires si la substitution de l'intérim à l'emploi en CDI se poursuit. La place des diplômes dans la sélection des ouvriers, et dans leur promotion en cas de recrutements en CDI, redeviendra alors d'actualité.

L'importance accordée à la détention d'un diplôme, au niveau et au contenu technique de celui-ci, dans la procédure de recrutement des ouvriers, fait l'objet de la première partie de cet article, tandis que la seconde est consacrée à l'impact du diplôme en termes de classification et de perspectives d'évolution.

\footnotetext{
${ }^{3}$ Dans la seconde moitié des années 90, les jeunes ouvriers sont très minoritaires à Peugeot-Sochaux. Une fois embauchés en CDI (contrat à durée indéterminée), "les jeunes veulent sortir au plus vite de leur poste en chaîne " (Hatzfeld, 2004 p. 28). Ils ont un $\mathrm{CAP}$, un BEP voire un bac professionnel ou technique, contrairement à la majorité des anciens, ce qui leur donne des atouts leur permettant de devenir polyvalents ou même moniteurs, au détriment des ouvriers plus âgés.
} 


\section{Encadré 1 \\ Les sources d'information}

Les informations ont été recueillies au cours d'enquêtes qualitatives sur le recrutement des ouvriers de la filière automobile effectuées dans la décennie 90 et dans la première moitié des années 2000, dans le cadre de conventions de recherche. Des entretiens auprès de directeurs ou de responsables des ressources humaines d'usines ont permis de revoir à plusieurs reprises les mêmes usines et de suivre ainsi les évolutions en matière de procédures de sélection. Seront ici principalement présentés les résultats de deux recherches financées par le ministère de l'Éducation nationale, dont l'objectif était d'analyser la relation formation-emploi, I'une en centrant l'analyse sur les diplômes de niveau V (CAP-BEP - certificat d'aptitude professionnelle et brevet d'études professionnelles) (Gorgeu, Mathieu, Pialoux, Blum, 2002) et l'autre sur les bacheliers (Blum, Gorgeu, Mathieu, 2005). La première visait à étudier les liens entre les pratiques de polyvalence et les exigences de diplômes ou de certifications pour les ouvriers. Elle a donné lieu, en 2000 et 2001, à une centaine d'entretiens auprès de responsables d'usines, de délégués syndicaux, de responsables d'ANPE (Agence nationale pour l'emploi), de professeurs et chefs de travaux de classes de CAP ou de BEP, de responsables d'organismes de formation continue, d'inspecteurs du travail, de responsables d'agences d'intérim, et de jeunes ayant préparé un CAP d'exploitant $d^{\prime}$ installations industrielles (EII)(*). Au total, 34 usines ont fait l'objet d'enquêtes : 6 usines de constructeurs et 28 usines fournisseurs. La seconde recherche, centrée sur les bacheliers utilisés comme ouvriers dans les usines d'équipement automobile, a porté sur 23 usines, et a donné lieu, en 2003 et 2004, à des entretiens auprès de directions d'usines d'équipement automobile, de conseillers d'ANPE, de responsables d'agences intérim, de jeunes ouvriers titulaires de baccalauréats, et de représentants syndicaux.

$\left(^{*}\right)$ : Renault est à l'origine de la création de ce CAP, en 1994, préparé à la fois en formation initiale et en formation continue (Gorgeu, Mathieu, 2002). Il a été remplacé en septembre 2003 par le CAP « Conduite de systèmes industriels ».

\section{LE DIPLÔME : UN PRÉ-REQUIS À L'EMPLOI QUI VARIE SELON LES USINES}

Un diplôme est nécessaire pour accéder à un emploi durable, voire même pour être intérimaire, mais les exigences de niveau et de spécificité de celui-ci sont différentes d'une usine à l'autre.

\section{Le diplôme, un préalable à l'embauche}

Un CAP ou un BEP est un préalable pour faire acte de candidature à un emploi d'opérateur ${ }^{4}$ dans les usines de la filière automobile. Dans le groupe

\footnotetext{
${ }^{4}$ Opérateur est le terme généralement utilisé pour les ouvriers de production considérés comme peu qualifiés (appelés aussi agents de production). Les professionnels sont des ouvriers hautement qualifiés qui peuvent être en production (cas des conducteurs d'installation), ou dans des fonctions annexes à la production, $\quad . . . / \ldots$
}

Renault, cette exigence est formalisée depuis 1998, mais ce diplôme de niveau V (diplôme de l'Éducation nationale ou titre AFPA - Association nationale pour la formation professionnelle des adultes - équivalent), peut concerner n'importe quelle spécialité. Il est suffisant en montage mais il ne l'est pas dans les usines de mécanique, où le baccalauréat professionnel en productique ou en maintenance est obligatoire. Chez PSA, l'usine Peugeot de Sochaux reconnait les certificats de qualification paritaires de la métallurgie (CQPM) comme équivalents aux diplômes de niveau V de l'Éducation nationale; en 2001, elle a d'ailleurs embauché en CDI des jeunes ayant obtenu en contrat de qualification des CQPM

.../... comme la maintenance. Les professionnels de maintenance peuvent être appelés aussi agents de maintenance. Les régleurs sont des ouvriers qualifiés qui, selon les usines, font partie de la production (c'est de la main-d'œuvre directe), ou sont dans des fonctions annexes (c'est de la main-d'œuvre indirecte). 
de "monteurs en ligne de fabrication automobile", « d'habilleurs moteurs », de « ferreurs » et de « peintres industriels ». Chez les fournisseurs, le pré-requis est en règle générale un CAP ou un BEP. Les propos de nos interlocuteurs montrent que sur les vingt-huit usines fournisseurs étudiées en 2000-2001, période d'expansion, seulement six, toutes situées en Franche Comté, n'appliquaient pas de critères de diplômes. Remarquons que plusieurs de ces usines n'utilisent pas non plus les tests. Certes, la localisation en Franche-Comté de ces usines et la reprise de l'emploi dans la région ont pu expliquer les moindres exigences de ces usines; cependant, il faut tenir compte du contenu du travail demandé, qui peut être simple (tri, rangement, manutention) en raison de l'activité de l'usine, ou des difficultés économiques qu'elle a pu connaître dans un passé récent. Cela peut être le cas notamment chez des fournisseurs de second rang. Ainsi, dans cette usine de traitement de surface, la responsable des ressources humaines ne demandait pas de diplômes: "On nous confie des pièces qu'on traite par zingage et par phosphatation. On a des opérateurs sur chaîne qui attachent des pièces sur des supports. Ils apprennent sur le tas. On sélectionne en fonction du physique, il faut être costaud et aussi grand, car les dernières chaînes dans lesquelles on a investi l'exigent. C'est un métier pénible où il faut être jeune. On ne peut pas être trop difficile. »

Le diplôme est appréhendé différemment selon les usines et les recruteurs. Un diplôme peut certifier un comportement. "Il garantit que le jeune est inscrit dans un cursus et est allé jusqu'au bout " (responsable emploi d'une usine de montage d'un constructeur), «Il valorise le jeune, et lui donne confiance en $l u i »$ (directeur des ressources humaines d'une usine fournisseur de plasturgie). Certains CAP de type artisanal, comme ceux de boucher, boulanger, pâtissier, cuisinier, sont appréciés parce que leurs titulaires sont considérés comme courageux, travailleurs, aptes à accepter des conditions de travail et d'horaires difficiles. Le diplôme peut jouer un rôle de garantie en matière de connaissances générales et de capacité d'évolution. Des responsables de ressources humaines ont insisté sur l'importance qu'ils accordaient à la rédaction et à l'orthographe, le diplôme de l'Éducation nationale avalisant, en principe, un niveau en français. Ainsi ce DRH (directeur des ressources humaines) d'une usine fournisseur à capitaux portugais, d'implantation récente, spécialisée en plasturgie, où il n'y avait que cinq personnes dépourvues de diplômes, donnait de l'importance à l'écriture : «Je regarde dans les CV les diplômes et l'expérience professionnelle, et j'écarte les $\mathrm{CV}$ et les lettres de motivation où il y a trop de fautes d'orthographe; il y a de l'écriture ici, ils ont des documents à écrire. La carence en français peut faire obstacle, être un frein. Le fait de ne pas bien écrire peut faire craindre que la personne soit un peu fruste. Ce n'est pas un ouvrier sur chaîne ici. Le gars regarde la pièce et a une responsabilité vis-à-vis de la production. Ils ont des feuilles de tâches et doivent faire une petite synthèse de leur travail à la fin de leur journée. » Pour cet autre interlocuteur, responsable emploi d'une usine de montage constructeur, les diplômes de l'Éducation nationale sont de meilleur niveau que les certificats de qualification professionnelle (CQP) car ils comportent des connaissances générales. "On fait du français quand on fait de la maintenance, car il faut savoir rédiger des comptes rendus; on fait aussi de la géométrie; on fait également du français en qualité. Le CAP doit donner plus de garanties en connaissances générales ». Le diplôme peut enfin être appréhendé pour les connaissances techniques qu'il apporte à son titulaire et, dans ce cas, ce sont des diplômes spécifiques qui sont privilégiés, en fonction des techniques utilisées, ou à défaut de type productique ou maintenance, certifiant des compétences en termes de polyvalence.

Il ressort des entretiens, en 2000 et 2001, que la possession d'un diplôme est nécessaire pour espérer accéder à un emploi durable. Nos recherches successives sur la gestion de la main-d'œuvre dans la filière automobile, depuis le début de la décennie 80 , témoignent d'une montée des exigences de niveaux de formation pour les ouvriers de production, qui va de pair avec une sélectivité accrue lors du recrutement ( $c f$. encadré 2). Un jeune qui sort du système scolaire avec une formation professionnelle mais sans diplômes est quasiment exclu par les constructeurs ; c'est moins vrai dans certains établissements d'équipement automobile. Dans ce cas, les emplois proposés, concurrencés par ceux des pays à 


\section{Encadré 2 \\ Le recrutement des opérateurs: la montée des exigences depuis une quinzaine d'années}

Dans les années 60 , les constructeurs automobiles ont fait venir des ouvriers immigrés qui ne savaient ni lire ni écrire. Il était relativement facile d'entrer sans qualification dans l'usine comme ouvrier de production dans les années 70 (Beaud, Pialoux, 2002). Nos enquêtes régulières auprès de responsables d'usines fournisseurs de l'automobile, au cours des décennies 80 et 90 , témoignent d'une montée ininterrompue de la sélectivité à l'embauche pour cette catégorie de personnel. L'émergence des normes qualité ISO et du référentiel commun d'assurance qualité fournisseur des constructeurs automobiles français en 1987(*) ont largement contribué à l'accroissement des exigences en termes de niveaux de formation. Le développement du chômage et des contrats précaires, notamment de l'intérim, la prolongation de la scolarité, l'objectif affiché par les Pouvoirs publics de $80 \%$ de bacheliers dans une classe d'âge, ont joué aussi un rôle important. À cet égard, nous nous sommes remémorés les propos d'un responsable du personnel d'une usine d'équipement automobile, située en pleine campagne dans la région Centre, visitée au cours de la deuxième moitié des années 80 (Gorgeu, Mathieu, 1990) : "Autrefois, dans le village, on disait aux enfants, si tu ne travailles pas bien à l'école, tu seras ouvrier chez M... Aujourd'hui, on leur dit si tu travailles bien à l'école, tu pourras peut-être être embauché chez M... »

Au début des années 90, les nouvelles usines d'équipement automobile, implantées à proximité des usines de montage des constructeurs, pour les livrer en juste à temps, sélectionnaient des jeunes ayant un niveau $V$ de formation (CAP-BEP - respectivement certificat d'aptitude professionnelle et brevet d'études professionnelles) pour être ouvriers de production. Le diplôme n'était pas obligatoire, et ils étaient évalués au cours d'un entretien, mais ils ne passaient pas de tests. En 1996-1997, dans les mêmes usines, le diplôme était exigé, au minimum un CAP ou un BEP, parfois un baccalauréat professionnel. En 2000 et 2001, malgré la diminution du chômage et l'apparition de difficultés de recrutement pour certains emplois dans certaines régions, les critères de sélection se sont renforcés pour les opérateurs avec la généralisation des tests. Les enquêtes réalisées en 2003 et 2004 montrent que, en quelques années, des transformations structurelles ont affecté la filière avec la création des Parcs Industriels Fournisseurs (PIF) ; ces derniers accroissent les exigences du juste-à-temps et la pression de l'usine de montage des constructeurs sur le personnel des équipementiers automobiles installés dans ces parcs, c'est-à-dire dans l'enceinte du site de montage du constructeur. Plusieurs responsables de ces usines nous ont précisé que le « savoir-faire gestuel » et la qualité étaient prioritaires, d'où l'importance accordée à l'expérience, aux résultats des batteries de tests et à la réputation des intérimaires. Des réflexions sont menées dans plusieurs entreprises sur l'articulation entre innovations technologiques et organisationnelles et compétences recherchées. Depuis longtemps, il était admis que l'automatisation, l'informatisation et l'instauration d'îlots autonomes de production entraînaient un accroissement de l'autonomie et de la qualification des opérateurs. Ce n'est plus une certitude pour toutes les équipes dirigeantes. Certains DRH (directeur des ressources humaines) reconnaissent s'être trompés, "avoir mis la barre de diplômes trop haute ". D'autres recherchent des CAP avec expérience, alors que ce diplôme était décrié il y a quelques années, ou préfèrent des personnes ayant le niveau baccalauréat: "Les opérateurs ayant un bac pro s'emmerdent. Je ne tiens pas à en avoir. Ceux qui ont le niveau bac pro n'ont pas la même mentalité car ils ont un sentiment d'échec. Je peux en prendre. » (DRH d'une usine de PIF en 2004)

$\left(^{*}\right)$ : Les normes internationales ISO 9000 d'assurance qualité, dont la première version date de 1987, sont destinées à homogénéiser à l'échelle mondiale les approches de la qualité des produits et des services. Elles n'ont pas suffi à Renault et PSA qui ont mis en place, en 1987, une démarche spécifique pour sélectionner leurs fournisseurs à partir d’un référentiel commun «Évaluation d'Aptitude Qualité Fournisseur ». 
bas salaires, sont à faible valeur ajoutée, mal payés, sans évolution possible.

L'enquête, en 2003-2004, sur les bacheliers ouvriers dans les usines d'équipement automobile révèle que dans la sélection d'un candidat en production et en logistique le diplôme ne joue plus le rôle de signal, comme c'était le cas au début des années 90. Aujourd'hui, les candidats sont sélectionnés par les agences d'intérim puis par les directions d'usines en cas d'embauche en CDD ou en CDI, voire pour tout prolongement d'une mission d'intérim ; à cet effet, est utilisée une série de tests vérifiant les connaissances et le comportement (Gorgeu, Mathieu, 2008). Est-ce le signe d'une méfiance vis-à-vis de la formation initiale des candidats? Parmi tous les candidats, il s'agit plutôt de sélectionner « les meilleurs ». Un diplôme, au minimum de niveau $\mathrm{V}$, est néanmoins un pré-requis pour les agences d'intérim, et donc pour les usines d'équipement automobile, puisque l'intérim est le canal exclusif de recrutement pour tout emploi d'opérateur. Les diplômes de niveau IV ne sont pas considérés comme véritablement différents de ceux de niveau V. Ils permettent d'occuper les mêmes emplois, mais leurs titulaires peuvent être jugés «à potentiel », c'est-à-dire capables d'évoluer. Avec le juste-à-temps ${ }^{5}$, il faut être rentable immédiatement, et s'adapter aux conditions de travail dans un temps très court. Cela dépasse la formation initiale. Ainsi il faut acquérir une expérience professionnelle pour être reconnu «à sa juste valeur » par les managers.

Les diplômes de l'Éducation nationale offrent plus de garanties aux entreprises que les autres certifications professionnelles. Celles-ci sont appréciées comme substituts aux diplômes en période de forte reprise de l'emploi, lorsque des pénuries de diplômés apparaissent, ce qui a été le cas en 2000-2001 en Franche Comté. Elles sont aussi considérées comme un complément à des diplômes généraux. Les usines de la filière automobile utilisent, en effet, de plus en plus les aides financières de l'État et de la région

\footnotetext{
${ }^{5}$ Le juste-à-temps est une méthode de pilotage des flux de l'aval vers l'amont qui permet de réduire les stocks. Les exigences de livraison en juste-à-temps des constructeurs à l'égard de leurs fournisseurs ont des conséquences importantes pour les fournisseurs qui sont incités à se rapprocher des usines clientes, à accrô̂tre la sélectivité à l'embauche, et à adopter une gestion de la maind'œuvre par les compétences.
}

pour former le personnel de production avant embauche définitive, ou pour se constituer un vivier d'intérimaires bien adaptés à leurs besoins. Cette possibilité de former à leurs techniques et à leurs méthodes des candidats sélectionnés, sans aucun engagement d'emploi, et ce à moindre coût, les incite à privilégier le niveau du diplôme, gage d'une aptitude à apprendre rapidement, plus que son contenu en connaissances techniques ${ }^{6}$.

\section{Le diplôme exigé varie selon les usines}

Pour des postes d'opérateurs, les diplômes professionnels et technologiques sont généralement recherchés, mais les baccalauréats généraux ou les diplômes supérieurs à ce niveau peuvent être néanmoins appréciés, car leurs titulaires sont considérés comme plus aptes à évoluer que des personnes moins diplômées. Pour les diplômes professionnels, le choix entre les niveaux IV et $V$ est subjectif ; il dépend de la personnalité du recruteur, de son âge ; ainsi, pour un même emploi de production dans des usines à activités identiques, des recruteurs privilégient des candidats ayant le baccalauréat professionnel quand d'autres acceptent des CAP ou BEP. La situation du marché du travail local interfère dans la prise de décision. En Franche Comté, avec la reprise économique de 1999 à 2001, les règles en matière de niveaux de diplômes se sont assouplies. S'agissant du contenu technique du diplôme, les demandes sont très variables d'une usine à l'autre, et il semble nécessaire de différencier les usines des constructeurs de celles des fournisseurs. Ces dernières ont des activités très variées et des produits spécifiques, n'utilisent pas les mêmes techniques, et n'ont pas les mêmes besoins en termes de compétences techniques pour leurs ouvriers de production. Les exigences peuvent être très générales, la préférence étant seulement accordée aux diplômes industriels ou à un champ plus réduit mais relativement large comme la mécanique; mais elles peuvent être aussi très précises, centrées sur un

\footnotetext{
${ }^{6}$ Des demandeurs d'emploi titulaires de baccalauréats technologiques de type tertiaire ou de baccalauréats généraux littéraires, de Deug (diplôme d'études universitaires générales), voire de licence ou de maîtrise, sont ainsi embauchés comme opérateurs classés non qualifiés, après avoir suivi des stages spécifiques dans le cadre de dispositifs de reclassement vers les métiers de l'industrie, destinés à des personnes « en difficultés d'emploi ».
} 
diplôme bien adapté au contenu du travail de l'opérateur ou choisi après expérimentation. Ainsi ce directeur des ressources humaines d'une usine fournisseur de plasturgie dans le Nord regrette qu'il n'y ait pas de lycée proche préparant des BEP plasturgie, mais a remarqué "que les opérateurs qui ont un BEP d'électrotechnique sortent du rang », et privilégie de ce fait ce diplôme. Par ailleurs, l'ANPE diffuse une méthode d'évaluation «des habiletés et des compétences $\gg{ }^{7}$ qui ne tient pas compte $a$ priori des diplômes, mais les directions d'entreprises qui l'utilisent observent que le taux de réussite des candidats s'élève en fonction du niveau d'études.

Les niveaux de diplômes exigés par les entreprises pour des postes d'ouvriers de production ou de professionnels (agent de maintenance, régleur, etc.) varient considérablement d'une entreprise à l'autre. Les entretiens auprès des salariés et des directions d'usines fournisseurs en 2003-2004 montrent une hétérogénéité de situations : des postes d'opérateurs occupés par des jeunes titulaires d'un diplôme de niveau $\mathrm{V}$ dans un autre domaine que celui de l'entreprise (CAP de boulanger, etc.), ou au contraire spécifiques à une technique recherchée par l'entreprise, des emplois d'opérateurs tenus par des bacheliers, alors que ce diplôme n'est pas exigé, mais aussi dans d'autres cas parce qu'il l'est. Quant aux postes d'agent de maintenance et de régleur, ils requièrent dans certaines entreprises un BTS (brevet de technicien supérieur) alors que des titulaires de baccalauréats "maintenance des systèmes mécaniques automatisés » (MSMA), « productique », « pilotage des systèmes de production automatisés » (PSPA) occupent ces fonctions dans d'autres. Le lien entre la formation et l'emploi peut subir des modifications dans une même entreprise, à des intervalles de temps rapprochés. Pour un même emploi, un directeur de ressources humaines peut exiger un baccalauréat

\footnotetext{
${ }^{7}$ Cette méthode a été élaborée en 2000 par l'ANPE, qui l'a d'abord expérimentée dans l'industrie automobile avant de la diffuser plus largement. Elle repose sur une batterie de tests visant à évaluer notamment l'aptitude à travailler en équipe selon des cadences définies et à maîtriser la qualité. La difficulté de ces tests semble s'être accrue d'après les propos d'un conseiller ANPE qui a utilisé cette méthode dans les mêmes usines automobiles en 2000 puis en 2003 : « ceux de contrôle sont plus pointus et ceux de rapidité et d'attention sont plus complexes. On les a réétalonnés à la demande de l'ANPE et des entreprises. »
}

pour un poste d'opérateur puis réduire ses exigences quelque temps après.

Si le pré-requis pour travailler en production ou en logistique est le CAP ou le BEP dans de nombreuses usines fournisseurs étudiées en 2003-2004, cette exigence des DRH n'exclut pas la possession de diplômes supérieurs. Nous pouvons identifier quatre manières d'employer les bacheliers dans ces usines :

\section{- Le baccalauréat n'est pas recherché en production}

Ce fonctionnement est fréquent dans les usines où la prégnance des coûts est particulièrement forte, c'està-dire dans les usines fournisseurs de montage où le travail manuel est prépondérant, mais aussi en plasturgie, même si les investissements y sont importants $^{8}$, et également dans toutes les usines situées à proximité immédiate des usines des constructeurs, notamment dans les usines des PIF. Ainsi la direction des usines ne recherche pas des bacheliers pour leurs emplois d'opérateurs. Ils entrent comme intérimaires ; ils peuvent ensuite passer en CDI sans que personne ne s'intéresse à leur diplôme. Celui-ci est passé inaperçu bien qu'il figure sur leur CV. Dans d'autres usines, le niveau de diplôme est connu et les titulaires de diplômes de niveau IV, voire supérieurs au bac, sans rapport avec l'activité de l'usine, sont prévenus qu'ils vont se déqualifier en acceptant d'occuper un emploi d'opérateur, et qu'ils n'ont aucune perspective de progression. Ce mode d'utilisation est fréquent dans des usines utilisant une main-d'œuvre ouvrière en majorité féminine, situées dans des bassins où les emplois tertiaires sont rares. Des jeunes femmes bachelières ou ayant des diplômes supérieurs acceptent ainsi une déqualification qui n'est pas toujours ressentie comme telle, car elles ne se font aucune illusion sur la valeur de leur diplôme. On retrouve les conclusions d'études de l'enquête "Génération 92 » du Céreq (Giret, Hatot, 2001) sur les facteurs explicatifs du déclassement subjectif et celles d'autres travaux (Baudelot, Gollac, 2002) qui montrent que les femmes jugent plus favorablement que les hommes leur situation sur le marché du travail.

\footnotetext{
${ }^{8}$ Cette activité est, rappelons-le, particulièrement concurrencée par les filiales de groupes équipementiers situés dans les pays à bas salaires.
} 


\section{- En production, une préférence pour des intéri- maires bacheliers}

Si les usines de ce groupe ont souvent les mêmes activités que les précédentes, elles s'en différencient par le fait que leur direction cherche à recruter ou à utiliser en intérim, en production, des bacheliers considérés comme plus performants, mais ce n'est pas dans le but de les faire évoluer. La déqualification des bacheliers est la même que dans les usines analysées ci-dessus. Plusieurs de ces usines n'embauchent plus en CDI et fonctionnent avec un volant permanent d'intérimaires. Ces usines sont situées, le plus souvent, dans des bassins d'emploi où le taux de chômage est très élevé. Les intérimaires représentent une part très importante de leur effectif en production. Plusieurs usines fournisseurs de montage et de plasturgie peuvent être classées dans cette catégorie. Une usine de fabrication de sièges située dans la région Nord à proximité immédiate de son client, qui fonctionne avec $400 \mathrm{CDI}$ et 350 intérimaires, a également un mode d'utilisation de ce type, même si elle prend en intérim à la fois des CAP-BEP et des bacheliers. Les propos de la responsable de recrutement montrent qu'elle souhaite plutôt des intérimaires titulaires de baccalauréats industriels : «On n'embauche plus en CDI, mais on prend en intérim comme opérateurs des gens ayant au minimum des CAP et des BEP dans le domaine industriel. Cela peut être quelqu'un qui sort de l'école. On prend aujourd'hui aussi des bacheliers professionnels sur les postes d'opérateurs. On a un implant de Vedior bis dans l'usine et on ne prend que des intérimaires ayant réussi leurs tests, qui sont difficiles. Les intérimaires sont partout. Au niveau intérim, s'il est au-dessus de BEP, CAP, même s'il a un BTS, cela ne pose pas de problèmes, bien au contraire. Les bacheliers professionnels restent opérateurs, et cela ne change rien pour l'individu d'avoir ce diplôme, car il fait le même travail que les autres.»

\section{- En production, le baccalauréat est recherché comme « potentiel » pour une éventuelle évolution}

Les directions de plusieurs usines recherchent des bacheliers comme opérateurs parce que leur objectif est de les faire progresser, ce qui n'est pas le cas dans le mode précédent, et qu'ils sont considérés comme plus aptes à évoluer. Mais les possibilités de promotion sont souvent très faibles parce qu'il n'y a pas de gestion prévisionnelle de la main-d'œuvre et pas de marché interne. Seules deux usines qui appartiennent à un même groupe canadien ont un véritable marché interne voulu par le groupe. Tous les postes à pourvoir font l'objet d'annonces internes; il faut postuler et réussir à un examen pour pouvoir évoluer. Les extraits d'entretien avec la DRH d'une de ces usines montrent que la détention d'un baccalauréat peut faciliter l'obtention de l'examen. L'expérience longue ainsi que la volonté peuvent aussi compenser le manque de diplômes, mais à compétences égales, le bachelier aura un parcours professionnel plus rapide. Dans cette usine, où le taux de formation a été très important $(10 \%$ de la masse salariale en 1999 et 2000 et $4 \%$ en 2003), un bachelier recruté comme opérateur peut espérer évoluer en postulant à un poste à pourvoir, s'il est prêt à investir en dehors de son temps de travail pour réussir l'examen, et cela lui sera plus facile s'il possède un baccalauréat carrosserie ou maintenance. Sa déqualification ne serait que provisoire. Cependant, cette usine n'embauche plus en CDI et a un faible taux de turnover, ce qui réduit les possibilités de promotion pour les opérateurs.

\section{- Les bacheliers sont embauchés à des postes qualifiés correspondant à leur formation}

Cinq usines d'équipement automobile, parmi celles analysées en 2002-2003, embauchaient des titulaires de baccalauréats techniques ou professionnels bien précis, notamment MSMA, comme ouvriers professionnels. Elles les utilisent le plus souvent comme conducteurs d'installation, emplois classés comme qualifiés, qui peuvent être dans certains cas occupés par des titulaires de BTS ${ }^{9}$. Le baccalauréat MSMA est en revanche, en général, jugé insuffisant pour un emploi de maintenance dans les usines d'équipement automobile. Une usine de fabrication de plaquettes de frein recrutait néanmoins des titulaires de ce diplôme comme professionnels de maintenance, et plusieurs jeunes bacheliers MSMA y exerçant cette fonction semblaient satisfaits de leur niveau de classification et de rémunération.

\footnotetext{
${ }^{9}$ Une DRH, nouvellement embauchée, venait de recruter trois bacheliers MSMA sur des postes de conducteurs d'installation car elle considérait que les BTS qui occupent aujourd'hui ces postes sont « surqualifiés » pour cet emploi.
} 
Le diplôme occupe une place importante dans le recrutement des ouvriers, même si les évaluations par tests et entretiens semblent aujourd'hui prioritaires, mais le niveau de diplôme exigé est variable selon les usines et peut changer au sein d'une même usine. Les quatre modes d'utilisation des bacheliers que nous avons distingués dans les usines fournisseurs montrent que ces bacheliers peuvent être utilisés comme opérateurs sans perspective de progression. Quel est l'impact du diplôme en termes de classification et d'évolution ? Il semble limité en règle générale, mais, là encore, notre analyse montre une grande diversité.

\section{L'IMPACT CONTRASTÉ} DU DIPLÔME SUR LA CARRIÈRE

\section{Le baccalauréat, un diplôme peu rémunérateur}

Pour des emplois d'agents de production et de logistique, la plupart des usines n'appliquent pas aux titulaires de diplômes de niveaux V et IV les coefficients de classification correspondant aux niveaux de diplômes prévus par les conventions collectives. En production, elles recrutent généralement au coefficient minimum ou immédiatement au-dessus, avec un salaire égal ou légèrement supérieur au Smic (salaire minimum $)^{10}$. L'embauche en CDI d'intérimaires améliore leur classement, car les intérimaires sont presque toujours classés au coefficient minimum de la convention collective, et il est très rare que leur situation soit revue lorsqu'ils cumulent plusieurs années d'ancienneté dans l'usine. La présence massive d'intérimaires entraîne ainsi une déqualification générale dans beaucoup d'usines. Les agences d'intérim, en pratiquant une forte sélection, peuvent proposer ces emplois de production prioritairement à des personnes possédant des diplômes de niveau IV, voire des diplômes supérieurs.

\footnotetext{
${ }^{10}$ Les caristes sont généralement mieux classés que les agents de production. Dans les usines qui adhérent à la convention collective de la métallurgie, ils sont au coefficient 170 (troisième coefficient après 145 et 155), qui est le premier correspondant à un emploi dit qualifié (P1). Pour un opérateur de production, devenir cariste est souvent considéré comme une promotion. Cette différence peut s'expliquer par le fait que les caristes doivent posséder le certificat d'aptitude à la conduite en sécurité (CACES) et qu'avec le développement des plates-formes logistiques, la demande est très forte.
}

La déqualification des bacheliers est plus importante chez les équipementiers que chez les constructeurs, ce qui peut s'expliquer par les exigences de réduction des coûts imposées par les constructeurs à leurs fournisseurs. Chez les constructeurs, les opérateurs de production sont embauchés, quel que soit leur diplôme, au coefficient 170 de la convention collective de la métallurgie, au lieu de 155 en règle générale chez les équipementiers relevant de cette convention. Par ailleurs, les constructeurs peuvent embaucher directement des bacheliers comme professionnels de maintenance ou conducteurs d'installation, ce qui est rare dans les usines d'équipement automobile ${ }^{11}$. Un bachelier professionnel utilisé au départ comme opérateur a de plus grandes chances d'évoluer dans une usine appartenant à un constructeur que chez un équipementier. Dans la plupart des usines fournisseurs étudiées en 2003-2004, la détention d'un diplôme de niveau IV ne permet pas à un ouvrier, qu'il soit en production ou en logistique, d'être classé à un coefficient supérieur à celui des autres ouvriers occupant les mêmes emplois mais n'ayant pas le même niveau de formation initiale.

C'est l'emploi occupé qui détermine le coefficient de classification et le salaire. En règle générale, à court terme, le baccalauréat n'apporte rien de plus aux jeunes, qu'ils soient intérimaires ou en CDI, qu'un CAP ou un $\mathrm{BEP}^{12}$. Dans quelques usines, néanmoins, les seuils d'accès en fonction des diplômes prévus par la convention collective sont pris en compte lorsque le baccalauréat correspond à l'emploi occupé ; mais le nombre de baccalauréats considérés comme adéquats peut être très faible; il semble en outre que la décision dépende de l'équipe dirigeante de l'usine, et soit prise en fonction de l'état du marché du travail local ( $c f$. encadré 3).

\footnotetext{
${ }^{11} \mathrm{D}$ 'après les propos des $\mathrm{DRH}$, certains baccalauréats industriels technologiques ou professionnels peuvent permettre d'accéder aux emplois d'ouvriers professionnels encore existants dans la filière automobile. Le baccalauréat technologique électrotechnique peut permettre d'être recruté comme conducteur d'installation, emploi considéré comme qualifié chez les constructeurs comme chez les équipementiers. Il en est de même du baccalauréat génie mécanique. Les baccalauréats professionnels installation d'équipements électriques, MSMA et productique, peuvent également conduire à ce métier.

${ }^{12}$ Une étude récente sur le déclassement tirée de l'enquête "Génération 98 » du Céreq (Lemistre, 2007) note un rapprochement entre les CAP-BEP et les bacheliers au niveau salaire, les écarts s'étant largement resserrés au regard de la « Génération 92 ».
} 
Le BTS, indispensable pour progresser?

La détention d'un diplôme de niveau IV facilite-telle l'évolution de carrière ? À cette question, il est difficile d'apporter une réponse simple et surtout généralisable.
Dans les usines enquêtées, les perspectives de promotion sont limitées pour les ouvriers de production ou de logistique. Pour les bacheliers, en règle générale, elles ne sont pas supérieures à celles des titulaires d'un CAP ou d'un BEP. Posséder un baccalauréat technologique ou professionnel industriel

\section{Encadré 3 \\ Quelques exemples d'interprétation de la correspondance entre formation et emploi}

Quatre usines d'équipement automobile étudiées en 2002-2003 exigent des baccalauréats techniques précis pour occuper des emplois qualifiés. Elles respectent les sevils d'accès de la convention collective de la métallurgie en fonction des diplômes pour leurs emplois de production, mais trois d'entre elles interprètent la correspondance diplôme-métier à leur façon. Une usine d'électronique embauche les CAP de I'industrie, les titulaires de titres (ministère du Travail) de l'AFPA (Association nationale pour la formation professionnelle des adultes), les titulaires de CQPM (certificats de qualification paritaires de la métallurgie), et les bacheliers généraux, au coefficient 155 de la métallurgie. Les BEP (brevet d'aptitude professionnelle) "dans nos métiers » (électronique, électrotechnique, maintenance et mécanique) sont classés au coefficient 170. Les bacheliers professionnels et technologiques "dans nos métiers » sont classés au coefficient 215. Cela a conduit la direction à réduire les exigences de diplômes pour un même emploi, lorsque l'usine a été contrainte, suite à une baisse de charges et à des impératifs de rentabilité, à comprimer ses coûts. Les bacheliers dans les métiers correspondant à l'activité de l'usine sont dorénavant considérés comme surqualifiés pour occuper des emplois où, un an auparavant, le baccalauréat était le minimum requis. Deux usines de découpage-emboutissage d'un même groupe, qui ont les mêmes activités et qui utilisent la grille de salaires élaborée au niveau du groupe, n'interprètent pas de la même manière la correspondance diplômes-métiers. Pour celle située en Franche Comté, il existe plusieurs catégories de conducteurs d'installation (Cl) dans l'usine: le $\mathrm{Cl}$ de presses automatiques, le $\mathrm{Cl}$ d'îlots d'assemblage et le $\mathrm{Cl}$ de lignes de presses robotisées (CI LPR) qui est un nouveau métier valorisé. C'est uniquement pour cette dernière catégorie de $\mathrm{Cl}$ qu'il existe une correspondance entre le diplôme et le coefficient, mais seuls les diplômes en électrotechnique sont reconnus : un titulaire de CAP-BEP électrotechnique occupant cet emploi est mis au coefficient 170, tandis qu'un bachelier en électrotechnique est classé au coefficient 190. Le principe dans cette usine est d'appliquer les seuils d'accès des conventions collectives uniquement quand il y a une correspondance stricte entre le diplôme et le métier. Dans l'autre usine, située en Basse Normandie, il y aurait une interprétation beaucoup moins restrictive des seuils d'accès de la convention collective en fonction des diplômes, comme le montrent les propos du responsable recrutement. "On respecte la grille conventionnelle de l'Orne, même si le bachelier exerce un emploi qui ne correspond pas à son diplôme. Un bac pro MSMA, productique, etc., à condition que ce soit dans la métallurgie, sera classé au coefficient 215 et aura le salaire associé à ce coefficient dans la grille de salaires du groupe. Les salaires de cette grille sont plus élevés que ceux de la convention collective de l'Orne. Ici, les organisations syndicales sont arrivées, lors des négociations salariales, à obtenir une correspondance entre niveau de diplômes et coefficient de classification, quel que soit le poste qui est tenu ". Cette situation semblait être la conjonction de trois faits. Cette usine était en expansion ; elle avait un syndicat majoritaire revendicatif et avait connu plusieurs grèves; elle n'était pas située dans une région métallurgique, et avait des difficultés, d'après notre interlocuteur, à embaucher de la maind'œuvre qualifiée en métallurgie. 
peut néanmoins accroître ses chances d'accéder à un emploi supérieur s'il y a une disponibilité. Mais le développement de la polyvalence et de la polycompétence peut constituer un frein à l'évolution; il est en effet fréquent que des ouvriers occupent provisoirement des emplois considérés comme plus qualifiés, sans reconnaissance en termes de classification et de salaire. La promotion n'est possible que si un poste se libère ou se crée.

Recruter des bacheliers sur des postes de production classés non ou peu qualifiés peut être un moyen de ne pas créer d'emplois de professionnels (ou d'en différer la création), et de demander à certains d'entre eux d'assumer des fonctions de professionnels sans en avoir le titre ni les avantages. Entre l'opérateur de production et les techniciens et agents de maîtrise, qui ont tous au moins un BTS, il n'existe bien souvent, surtout en montage, que l'emploi 《d'animateur $»^{13}$, poste à responsabilités ne rentrant pas dans la hiérarchie de l'entreprise. Les animateurs, en nombre restreint, sont des opérateurs «plus » qui ne sont guère mieux rémunérés que les autres. Le diplôme n'est pas déterminant dans le choix des opérateurs nommés «animateurs", comme le montrent ces propos d'une responsable recrutement dans une usine de fabrication de sièges : «Sur 12 jeunes ayant préparé en contrats d'apprentissage chez nous un bac pro, on en a pris seulement 2, ceux qui l'avaient obtenu avec mention ou qui étaient les moins absents; ce sont des gens à potentiel qui occupent les mêmes postes que les CAP-BEP. On les a mis comme opérateurs. La seule perspective d'évoluer, c'est de devenir animateur. On ne nomme pas les opérateurs comme animateurs en fonction de leur diplôme. Un bac pro peut être moins bon qu'un CAP. En fait, on prend volontairement comme animateurs ceux qui s'efforcent de progresser, quel que soit leur diplôme. »

Le diplôme peut permettre d'évoluer dans les usines d'équipement automobile qui embauchent des bacheliers sur des postes qualifiés ou qui les forment dans ce but - mais elles étaient peu nombreuses en 20032004 -, et dans celles qui ont un marché interne, cas

${ }^{13}$ Nous utilisons ce terme par commodité, car son titulaire a pour rôle d'exercer une fonction d'animation de son équipe tout en assumant la production; les appellations varient d'une usine à l'autre (« animateur », « moniteur », « régulateur », « team leader », etc.). rarissimes. La plupart des jeunes bacheliers se retrouvent en production sans perspective d'amélioration de leur situation. Le travail de production dans cette industrie ne mobilise pas les compétences acquises en formation initiale ; il est jugé routinier, inintéressant, même si l'opérateur doit assumer, en plus de la production, des fonctions en matière de qualité, maintenance de premier niveau, gestion de production. Quasiment tous jugent que la détention d'un baccalauréat professionnel n'est pas nécessaire pour les emplois proposés car les tâches qui requièrent, de leur point de vue, une réelle qualification occupent une trop faible part de leur temps. La référence du travail vraiment qualifié est pour eux le travail artisanal ou la fonction maintenance. Avec la production en grandes séries, le travail reste répétitif, ce qui explique l'insatisfaction des jeunes bacheliers professionnels, davantage attirés par la production à l'unité ou en petites séries, mais qui ont été contraints d'accepter ces postes ( $c f$. encadré 4). Ils ont l'impression que tout le monde pourrait exercer ces activités, et que dans ces usines, leur formation technique est un investissement perdu. Si des tâches d'assistance à la production relatives à la qualité, à la maintenance, à la gestion de production, voire au management, dans le cas des animateurs, leur sont dévolues, elles ne sont qu'annexes car il leur faut d'abord produire dans l'urgence. La compétition est vive néanmoins entre les opérateurs pour occuper les nouveaux emplois de production comportant des tâches plus qualifiées; ils apportent certes peu d'avantages en termes de qualification et de rémunérations, mais ils sont les seuls accessibles, puisque nombre d'emplois indirects ont disparu ou sont réservés dorénavant à des titulaires de BTS ou de diplômes d'ingénieurs.

Les jeunes bacheliers qui occupent des emplois d'ouvriers non qualifiés ne se font guère d'illusions sur leurs possibilités de promotion. Les baccalauréats professionnels ne permettent pas aujourd'hui d'accéder, pour des jeunes sur le marché du travail, à des emplois "hautement qualifiés » dans le monde industriel tel qu'il est spécifié dans le référentiel de l'Éducation nationale. Il n'y a pas d'uniformité dans la gestion du personnel parmi les usines de la filière automobile. Avec le même baccalauréat professionnel industriel, par exemple le baccalauréat MSMA, un 


\section{Encadré 4}

\section{Pourquoi des bacheliers acceptent-ils des emplois non qualifiés?}

Les jeunes bacheliers professionnels, occupant des emplois peu qualifiés dans les usines d'équipement automobile, sont insatisfaits de ne pas utiliser les connaissances acquises, et jugent leur rémunération insuffisante. Ils acceptent ces emplois car ils ont intériorisé la dévalorisation des diplômes professionnels de niveau inférieur au BTS (brevet de technicien supérieur). Leur origine sociale explique en grande partie cette acceptation, leurs parents ne disposant pas de revenus et de réseaux leur permettant de continuer leurs études, ou de refuser les emplois ne correspondant pas à leur formation, notamment lorsqu'ils sont en intérim. Cette acceptation peut aussi s'expliquer par leurs difficultés d'insertion à la sortie de formation. Des trajectoires professionnelles marquées par le chômage et la précarité conduisent à ne pas être difficile, à prendre toutes les missions proposées par les agences d'intérim, à se montrer docile dans l'usine pour obtenir un CDI (contrat à durée indéterminée) ou être rappelé pour une nouvelle mission. S'ils restent intérimaires, ils n'ont aucune chance de progresser, et quand ils occupent momentanément un poste considéré comme qualifié, ils peuvent continuer à être au coefficient le plus bas et à ne pas être mieux rémunérés. Ceux qui sont surqualifiés, en raison de leurs diplômes, par rapport aux postes qu'ils occupent en production, sont parfois prêts, pour sortir de la précarité, à accepter en CDI un emploi non qualifié avec l'espoir de progresser ensuite. Mais le pourrontils? Analysant la trajectoire des jeunes passés par un emploi non qualifié, dans le cadre de l'enquête "Génération 98 » du Céreq, Marie-Odile Lebeaux (2004) observe qu'avoir travaillé comme employé non qualifié et comme ouvrier non qualifié caractérise des parcours qui ne permettent que très rarement et difficilement de sortir de l'emploi non qualifié.

jeune peut travailler comme opérateur non qualifié dans une usine et comme professionnel de maintenance dans une autre. La détention d'un BTS industriel est généralement indispensable pour pouvoir « sortir» de la production et évoluer, surtout chez les équipementiers qui exigent fréquemment ce diplôme pour accéder à des emplois de professionnels de maintenance. Il semble que dans les groupes fournisseurs, le management ait fait la même erreur que les équipes dirigeantes des constructeurs quelques années auparavant lorsqu'ils ont recruté pour leurs ateliers de montage des bacheliers professionnels. La «barre » a été mise trop haute pour des emplois aux tâches routinières et sans perspectives d'évolution. Parmi les jeunes bacheliers interrogés en 2003, quelques-uns, qui ne sont pas parvenus à obtenir un contrat à durée indéterminée plusieurs années après leur sortie de formation initiale, pensent ainsi que leur baccalauréat professionnel correspond à un niveau intermédiaire qui ne devrait pas conduire directement à la vie active. Ils considèrent qu'il est préférable soit de s'arrêter au BEP, soit de poursuivre en obtenant un BTS. Ils s'appuient sur l'expérience d'une ou deux personnes qui ont travaillé à la sortie de leur BEP (diplôme qu'ils ont préparé ensemble) et qui occupent, au moment de l'entretien, une position plus favorable que la leur.

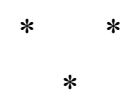

L'analyse des modes d'utilisation de diplômés de niveaux V et IV (CAP-BEP et baccalauréat) sur des postes d'ouvriers dans les usines de la filière automobile révèle des situations contrastées. Des différences dans l'importance accordée au niveau et au contenu technique du diplôme et dans la prise en compte de celui-ci dans la classification et les perspectives d'évolution des ouvriers apparaissent entre constructeurs et fournisseurs, mais aussi, chez ces derniers, d'un établissement à l'autre. Les directions d'usines sont très pragmatiques et leur marge d'autonomie est limitée ; elles sont en effet tributaires des orientations prises au niveau du groupe en matière de réduction 
des coûts. Ainsi, les entreprises de la filière automobile ont des pratiques de gestion de l'emploi et de la main-d'œuvre qui leur sont propres, avec notamment un recours très important à l'intérim. Avec la banalisation de ce dernier, le rôle « protecteur » du diplôme contre la déqualification se réduit, ce constat corroborant les résultats plus généraux de l'enquête « Génération 92 » du Céreq (Arliaud, Eckert, 2002).

Le baccalauréat ne permet pas aux jeunes qui entrent comme ouvriers dans les usines de cette filière de valoriser leurs connaissances. Il leur confère un « potentiel» qui devrait leur permettra de progresser en fonction de leurs compétences, encore faut-il que l'entreprise leur offre réellement cette possibilité. Cette évolution est perturbée par le raccourcissement de la ligne hiérarchique, la réduction des emplois d'assistance à la production, la compression des budgets formation, et la gestion par les compétences « qui aboutit à un système où chacun est jugé par son supérieur hiérarchique», et où "quand l'inné interfère avec la qualification, la voie est ouverte à l'arbitraire le plus total » (Cornu, Eckert, Doazan, 2002).

La réhabilitation du CAP, à la fin des années 90, la multiplication des spécialités de baccalauréats professionnels ${ }^{14}$, le développement des certifications répondent à une nouvelle priorité qui est de conduire cent pour cent d'une classe d'âge à un diplôme ou à un titre professionnel. Se pose néanmoins le problème de la sélectivité des diplômes professionnels, qui, d'après

${ }^{14}$ En 2006, la liste officielle des diplômes publiée par le ministère de l'Éducation nationale recense 213 CAP, 35 BEP, 69 baccalauréats professionnels, 62 brevets professionnels et 113 BTS (Maillard, 2006a).
Fabienne Maillard (2006b), est un élément constitutif de ces derniers ${ }^{15}$.

Une autre question récurrente est celle de la reconnaissance des diplômes et autres titres professionnels. José Rose (2006) souligne que les diplômes et certificats sont pour les employeurs des « réducteurs d'incertitude », mais que rien ne garantit que cela leur confère de la valeur. Les certifications ne sont pas reconnues dans les conventions collectives, contrairement aux diplômes, et dans les entreprises les coefficients de classification correspondant aux niveaux de diplômes ne sont que rarement appliqués. Nous le constatons dans la filière automobile, mais le phénomène est plus général. Comme le note Emmanuel Quenson (2006), en France, rien n'engage les entreprises à le faire, contrairement à l'Allemagne, où les diplômes et autres certifications permettent l'accès à une qualification identifiée. En outre, cet auteur constate que l'absence de perspectives professionnelles est particulièrement nette pour les diplômes transversaux, comme le baccalauréat «Pilotage de systèmes de production automatisés », qui ont été créés pour répondre à la demande d'industriels soucieux de disposer rapidement d'une main-d'œuvre formée, réactive, et rapidement autonome. Ainsi des formations qui profitent directement aux employeurs conduisent souvent au blocage des jeunes dans des emplois d'exécution sans réelles perspectives de mobilité professionnelle ascendante.

15 «Inégale selon les diplômes, selon les spécialités, mais aussi selon les époques, la sélectivité des évaluations fait en tout cas des diplômes professionnels les moins accessibles de tous les diplômes de l'Éducation nationale» précise Fabienne Maillard (2006b p. 140) qui note qu'en 2005 les taux de réussite étaient de $77 \%$ en CAP, $75 \%$ en baccalauréat professionnel, et de $66 \%$ en BTS. 
Arliaud M., Eckert H. (2002), Quand les jeunes entrent dans l'emploi, La Dispute.

Baudelot C., Gollac M. (2002), Travailler pour être heureux? Le bonheur et le travail en France, Fayard, Paris.

Baudelot C., Leclercq F. (Dir.), (2005), Les effets de l'éducation, Paris, La Documentation française.

Beaud S., Pialoux M. (2002), «Changements dans les rapports entre générations ouvrières - les années 90 à Sochaux-Montbéliard », Retraite et Société, $\mathrm{n}^{\circ} 35$, janvier.

Blum O., Gorgeu A., Mathieu R. (2005), Les bacheliers dans les usines d'équipement automobile. Vers la déqualification? ministère de l'Éducation nationale, CPC Documents ${ }^{\circ} 1$.

Cornu R., Eckert E., Doazan Y. (2002), « Retour sur la relation formation-emploi », Formation Emploi, $\mathrm{n}^{\circ} 78$.

Eckert H. (2006), Avoir vingt ans à l'usine, La Dispute.

Giret J.-F., Hatot C. (2001), « Mesurer le déclassement à l'embauche: l'exemple des DUT et des BTS », Formation Emploi, $\mathrm{n}^{\circ} 75$.

Gorgeu A., Mathieu R. (1990), « Partenaire ou soustraitant?», Dossier $n^{\circ} 31$ du Centre d'Études de l'Emploi, Noisy-le-Grand.

Gorgeu A., Mathieu R. (2002), «Le CAP "Exploitation d'installations industrielles": une seconde chance pour les non-diplômés?», Formation Emploi, $\mathrm{n}^{\circ} 80$.

Gorgeu A., Mathieu R. (2008), « La déqualification ouvrière en question », Formation Emploi, n 103.

Gorgeu A., Mathieu R., Pialoux M., Blum O. (2002), La polyvalence ouvrière dans la filière automobile: exigences et pratiques, ministère de l'Éducation nationale, CPC/Documents 2002/3.

Hatzfeld N. (2004), «L'individualisation des carrières à l'épreuve. Les grippages de la mobilité sur les chaînes de Peugeot-Sochaux ", Sociétés Contemporaines $\mathrm{n}^{\circ} 54$.

Lebeaux M.-O. (2004), « Un emploi non qualifié en début de carrière et après ? ", Document de travail, Céreq, collection «Net-Doc », $\mathrm{n}^{\circ} 4$, avril.

Lemistre P. (2007), « Un déclassement surévalué aux causes et conséquences sous-estimées? », Note $d u$ LIRHE 443 (07-6), février.

Maillard F. (2006a), « Les diplômes professionnels dans l'espace des certifications. Un rôle et une place en évolution », in Maillard F. (Dir.), Les diplômes de l'Éducation nationale dans l'univers des certifications professionnelles, nouvelles normes et nouveaux enjeux, CPC Documents n 3 .

Maillard F. (2006b), « Les diplômes professionnels face à la nouvelle norme certificative : ajustements, contradictions et tensions ", in Maillard F. (Dir.), Les diplômes de l'Éducation nationale dans l'univers des certifications professionnelles, nouvelles normes et nouveaux enjeux, CPC Documents $\mathrm{n}^{\circ} 3$.

Merle V. (2002), La démocratisation de l'enseignement, La Découverte, « Repères ».

Quenson E. (2006), « La transversalité des diplômes de la production : un modèle de certification reconnu sur le marché du travail », in Maillard F. (Dir.), Les diplômes de l'Éducation nationale dans l'univers des certifications professionnelles, nouvelles normes et nouveaux enjeux, CPC Documents $\mathrm{n}^{\circ} 3$.

Rose J. (2006), «Diplômes et certifications - les termes du débat, les lignes d'un programme de recherches », in Maillard F. (dir.), Les diplômes de l'Éducation nationale dans l'univers des certifications professionnelles, nouvelles normes et nouveaux enjeux, CPC Documents $n^{\circ} 3$. 


\title{
Résumé La place des diplômes dans la carrière des ouvriers de la filière automobile
}

\author{
Armelle Gorgeu et René Mathieu
}

La sélection à l'embauche des ouvriers de la filière automobile (constructeurs et fournisseurs) repose sur une succession de filtres où les évaluations par tests et entretiens semblent prioritaires. Néanmoins, le diplôme occupe une place importante dans les recrutements. Selon les usines et les recruteurs, il certifie un comportement, est garant de connaissances générales et de capacité d'évolution, voire de compétences techniques. Pour tout emploi d'ouvrier, même en intérim, un diplôme de niveau V (CAP/ BEP - certificat d'aptitude professionnelle et brevet d'études professionnelles), est ici un pré-requis. Ceux de niveau IV (baccalauréat) permettent d'occuper les mêmes emplois et peuvent faciliter l'évolution professionnelle, mais en règle générale, seul le BTS (brevet de technicien supérieur) permet de progresser. La déqualification des ouvriers bacheliers est plus importante chez les fournisseurs que chez les constructeurs automobiles.

\section{Mots clés}

Industrie automobile, critère d'embauche, niveau de formation, cheminement professionnel Journal of Economic Literature: M 51, J 23 